\title{
Bibliometric mapping of computer and information ethics
}

\author{
Richard Heersmink • Jeroen van den Hoven • \\ Nees Jan van Eck $\cdot$ Jan van den Berg
}

Published online: 19 April 2011

(c) The Author(s) 2011. This article is published with open access at Springerlink.com

\begin{abstract}
This paper presents the first bibliometric mapping analysis of the field of computer and information ethics (C\&IE). It provides a map of the relations between 400 key terms in the field. This term map can be used to get an overview of concepts and topics in the field and to identify relations between information and communication technology concepts on the one hand and ethical concepts on the other hand. To produce the term map, a data set of over thousand articles published in leading journals and conference proceedings in the C\&IE field was constructed. With the help of various computer algorithms, key terms were identified in the titles and abstracts of the articles and co-occurrence frequencies of these key terms were calculated. Based on the co-occurrence frequencies, the term map was constructed. This was done using a computer program called VOSviewer. The term map provides a visual representation of the C\&IE field and, more
\end{abstract}

R. Heersmink $(\varangle) \cdot$ J. van den Hoven

Philosophy Section, Delft University of Technology, Delft,

The Netherlands

e-mail: richard.heersmink@gmail.com

J. van den Hoven

e-mail: m.j.vandenhoven@tudelft.nl

R. Heersmink

Macquarie Centre for Cognitive Science, Macquarie University,

Sydney, NSW, Australia

N. J. van Eck

Centre for Science and Technology Studies, Leiden University, Leiden, The Netherlands

e-mail: ecknjpvan@cwts.leidenuniv.nl

J. van den Berg

Information and Communication Technology Section,

Delft University of Technology, Delft, The Netherlands

e-mail: j.vandenberg@tudelft.nl specifically, of the organization of the field around three main concepts, namely privacy, ethics, and the Internet.

Keywords Bibliometric mapping - Computer and information ethics - Term map · VOSviewer

\section{Introduction}

Computer and information ethics (C\&IE) is a branch of applied ethics that emerged in the 1980s. It studies the social and ethical impact of information and communication technology (ICT) on individuals and society. The more specific term 'computer ethics', coined by Walter Maner in the 1970s, refers to the application of normative theories such as utilitarianism, Kantianism, and virtue ethics to particular ethical cases that involve computer systems or networks. Computer ethics is also used to refer to professional ethics for computer professionals, such as ethical codes of conduct that can be used as guidelines for ethical cases. In 1985, Jim Moor (1985) and Deborah Johnson (1985) published seminal texts that helped define the field of C\&IE. From then on, C\&IE was recognized as an established field in applied ethics with its own journals, conferences, research centers, and professional organizations. Particularly in the last decade, ICT received a significant amount of attention from ethicists as well as sociologists, anthropologists, and scholars in law, education, and communication studies. Recently, computer ethics broadened to ethics of information technology and information ethics, a more general field that includes computer ethics, media ethics, library ethics, and bioinformation ethics (Brey and Søraker 2009).

In recent years, a number of attempts were made to provide overviews of the complex field of C\&IE. These 
overviews can be found in handbooks (Floridi 2010; Johnson 2008; Tavani and Himma 2008; van den Hoven and Weckert 2008), encyclopedia articles (Brey and Søraker 2009; Bynum 2008), and papers (Buchanan 1999). These sources give overviews of the C\&IE field by providing a historical sketch of the field and by discussing and analyzing the most important topics.

In this paper, we also aim to provide an overview of the C\&IE field. However, the approach that we take is different from previous approaches. In contrast to earlier work, we take a bibliometric mapping approach. ${ }^{1}$ This approach is data driven and relies heavily on computer algorithms and visualization techniques. The approach can be seen as complementary to the earlier studies mentioned above. The main result of our bibliometric mapping approach is a term map of the C\&IE field. This map provides a visual representation of the field by showing the relations between 400 key terms in the field. The term map, produced using the VOSviewer software for bibliometric mapping (Van Eck and Waltman 2010), is based on the titles and abstracts of over 1,000 articles that were published in leading journals and conference proceedings in the C\&IE field in the period 2003-2009. The map can be used to get an overview of concepts and topics in the field and to identify relations between ICT concepts (e.g., Internet, ambient intelligence, robotics) on the one hand and ethical concepts (e.g., privacy, autonomy, freedom) on the other hand. Based on the term map, we provide an analysis of the C\&IE field. In our analysis, the term map will be guiding, but we will also rely heavily on our own expertise in the field in order to give a proper interpretation to the map.

The rest of this paper is organized as follows. We focus on our methodology in "Methodology". We first discuss how we constructed our data set, and we then explain how we performed our bibliometric mapping analysis. We present our results in "Results". Finally, in "Conclusions", we summarize our main findings and we draw some general conclusions.

\section{Methodology}

\section{Data set}

The first step in this study is the construction of a representative data set of C\&IE literature. To construct this data set, we needed to select the sources in which the most

\footnotetext{
$\overline{1}$ Our paper is the first study in which a bibliometric mapping approach is used to analyze the C\&IE field. Our methodology has also been briefly discussed in Stahl et al. (2010). A somewhat related approach was taken by Ocholla et al. (2010). These authors used bibliometric techniques to study the concept of information ethics. However, they did not produce bibliometric maps.
}

important studies on ethical issues related to ICT can be found. Scholars in the C\&IE field publish and interact in a limited number of journals and conferences. We selected these journals and conferences based on our experience and expertise in the field and also based on information provided by journals and conferences on their websites. After selecting the relevant journals and conferences, we constructed an extensive data set. This data set consists of titles and abstracts of articles published in 12 journals and 3 conference proceedings. Because we did not have access to the full texts of all articles, we decided to analyze only titles and abstracts. We do not see this as a significant limitation. Titles and abstracts are supposed to represent the full contents, including key concepts, of an article, and supposedly titles and abstracts are more focused than full texts and therefore more suitable for automated analysis. We only considered articles that were published in the period 2003-2009. This period is representative for the recent development of the C\&IE field. During this period, the field has grown substantially and has become more mature. Also, the number of journals in the field has increased considerably during this period. Of course, the C\&IE field goes back further than 2003, but our aim is to focus on the recent development of the field rather than on its earlier development.

The following 6 journals explicitly deal with ethical issues of information and ICT. For each journal, we indicate the issues from which articles were taken.

- Ethics and Information Technology From volume 5, issue 1 to volume 11, issue 4 (161 articles; period 2003-2009).

- Information, Communication and Society From volume 6 , issue 1 to volume 12, issue 7 (235 articles; period 2003-2009).

- International Review of Information Ethics (formerly known as International Journal of Information Ethics). From volume 1 to volume 11 (120 articles; period 2004-2009).

- Journal of Information, Communication and Ethics in Society From volume 1, issue 1 to volume 7, issue 4 (121 articles; period 2003-2009).

- Journal of Information Ethics From volume 15, issue 1 to volume 18, issue 1 (27 articles; period 2006-2009).

- The Ethicomp Journal From volume 1, issue 1 to volume 3, issue 2 (127 articles; period 2004-2008).

Because of the convergence of ICT with other technologies, such as biotechnology, nanotechnology, and cognitive science, we also included articles from journals that cover the phenomenon of converging technologies. Furthermore, in addition to the journals listed above, there are other journals that also publish articles on C\&IE. We selected the following 6 journals that are relevant and 
leading in fields adjacent to C\&IE. From these journals, we only considered articles that are related to ICT.

- $A I$ \& Society From volume 17, issue 1 to volume 24, issue 4 (50 articles; period 2003-2009).

- Behavior and Information Technology From volume 22 , issue 1 to volume 28, issue 6 ( 8 articles; period 2003-2009).

- Nanoethics From volume 1, issue 1 to volume 3, issue 2 (1 article; period 2007-2009).

- Neuroethics From volume 1, issue 1 to volume 2, issue 3 (4 articles; period 2008-2009).

- New Media and Society From volume 5, issue 1 to volume 11, issue 6 (62 articles; period 2003-2009).

- Science and Engineering Ethics From volume 9, issue 1 to volume 15, issue 3 (18 articles; period 2003-2009).

There are several conferences that are partially or completely devoted to C\&IE. These conferences have proceedings in which conference articles are published. We selected the following two conferences. In the case of the Society for Philosophy and Technology conference, only articles from the Philosophy and Ethics of Information Technology track were considered.

- Computer Ethics Philosophical Enquiry (75 articles; 2005 and 2007).

- Society for Philosophy and Technology (18 articles; 2009).

In total, 1,027 articles were collected from the journals and conferences listed above. Our data set consists of the titles and abstracts of these articles. The titles and abstracts were retrieved manually from the websites of the journals and conferences.

A number of possible limitations of our data set should be mentioned. First, in our selection of journals and conferences, we may have missed some relevant sources. Second, the data set is based on English language articles only. Articles in other languages have not been considered. Third, apart from journals and conferences, other academic sources, such as (edited) books, encyclopedias, course materials, and reading lists, have not been considered. Fourth, non-academic sources that also discuss ethical issues related to ICT have not been considered. Examples of such sources include newspapers, newsletters, websites, and blogs. It follows from these limitations that our analysis is entirely focused on English language, peer reviewed academic literature.

\section{Bibliometric mapping}

We analyzed our data set using a bibliometric mapping approach. Bibliometrics is the scientific field that is concerned with the quantitative analysis of books, articles, and other types of written communication. In the field of bibliometrics, a significant amount of attention is paid to bibliometric mapping. Bibliometric mapping aims to produce visual representations of the relations between certain units of interest. The units of interest can be for example documents, authors, or keywords, and the relations between the units can be based on for example citations, co-citations, co-authorships, or co-occurrences of keywords. In our analysis, we focus on relations between key terms in C\&IE based on co-occurrences in titles and abstracts of articles. We refer to the map that we produce as a term map. The bibliometric literature on this type of maps goes back to the 1980s (e.g., Callon et al. 1986; Peters and Van Raan 1993; Tijssen and Van Raan 1989). In the literature, the maps are often referred to as co-word maps. We will now discuss our bibliometric mapping approach in more detail.

Using a computer algorithm (Van Eck et al. 2010, pp. 584-585), we identified noun phrases in the titles and abstracts of the 1,027 articles in our data set. For our purpose, we define a noun phrase as a sequence of words such that the last word in the sequence is a noun and each other word is either a noun or an adjective. Only noun phrases occurring at least 6 times in the titles and abstracts were taken into consideration. This resulted in a set of 1,368 noun phrases. Some noun phrases, such as Author, Abstract, or Preparation, are not particularly informative about C\&IE. We manually identified 661 of these irrelevant noun phrases. The irrelevant noun phrases were excluded from further analysis. Out of the remaining 707 noun phrases, the 400 noun phrases that seemed most relevant and most interesting were selected. The selection of the 400 noun phrases was done using an automatic term identification technique (Van Eck et al. 2010). This technique aims to distinguish general noun phrases with a broad meaning (e.g., Need, State, and Quality) from more specific noun phrases (e.g., Informational Privacy, Teaching Ethics, and Computer Professional). The latter noun phrases tend to be the more interesting ones, and these noun phrases were therefore selected. The selected noun phrases can be regarded as important terms in C\&IE. The rest of our analysis is based on the selected set of 400 terms. $^{2}$

For each pair of terms, we counted the number of times the terms occur together in the same article (or more precisely, in the title or abstract of the same article). Co-occurrence frequencies of terms are a commonly used

\footnotetext{
${ }^{2}$ We also tried to do our analysis with all 707 noun phrases that remained after the 661 irrelevant noun were removed. However, due to the presence of a significant number of quite general noun phrases, this did not yield satisfactory results. We therefore decided to make a further selection of noun phrases using the automatic term identification technique.
} 
measure of the relatedness of terms. We used the cooccurrence frequencies of our terms as input for a computer program called VOSviewer (Van Eck and Waltman 2010; freely available at http://www.vosviewer.com). Based on the co-occurrence frequencies, the VOSviewer software constructed a term map. This is a 2-D map in which the 400 terms are located in such a way that the distance between any two terms reflects the relatedness of the terms as closely as possible. In general, the stronger the relation between two terms, the smaller the distance between the terms in the map. Each term in the term map also has a color. Colors are used to indicate the grouping or clustering of the terms. Terms with the same color belong to the same cluster and tend to be more closely related than terms with different colors. In other words, terms with the same color tend to co-occur with each other more frequently than terms with different colors. The clustering consists of three clusters. We also tried out larger numbers of clusters, but a term map with three clusters seemed to yield the most satisfactory interpretation.

To obtain the mapping and the clustering of the terms, the VOSviewer software uses two techniques, namely the VOS mapping technique and the VOS clustering technique. VOS is an acronym for visualization of similarities, where in our case the similarities were given by the co-occurrence frequencies of the terms. The VOS mapping technique and the VOS clustering technique share the same underlying principles, and together these techniques provide an unified framework for mapping and clustering (Waltman et al. 2010). The VOS mapping technique is closely related to the well-known statistical technique of multidimensional scaling (e.g., Borg and Groenen 2005). We refer to Van Eck et al. (2010) for a discussion of the advantages of the VOS mapping technique over traditional multidimensionalscaling-based approaches to bibliometric mapping. The VOS clustering technique is closely related to clustering techniques that are based on the so-called modularity function of Newman and Girvan (2004; see Waltman et al. 2010). These techniques are very popular in the physics literature. Unlike standard modularity-based clustering techniques, the VOS clustering technique has a resolution parameter that determines the level of detail of the clustering that is produced. As mentioned above, we have chosen to work with a relatively low level of detail, resulting in only three clusters.

\section{Interpretation of a bibliometric map}

The interpretation of a bibliometric map is not entirely straightforward. Because bibliometric mapping has a number of limitations, the interpretation of a map should always be done in a very careful manner. Essentially, there are two types of limitations of bibliometric mapping, namely limitations imposed by the data and limitations imposed by the map:

- Limitations imposed by the data The availability of data will always be limited, and the data that is available will always contain a certain amount of noise. In our case, noise in the data may arise from the somewhat arbitrary decisions researchers make when choosing the terminology they use in the titles and abstracts of their articles. Researchers may also use synonyms and homonyms. Our techniques do not recognize synonyms and homonyms, and this may also make the interpretation of our results somewhat ambiguous.

- Limitations imposed by the map A map provides a simplified representation of reality, and simplification generally implies some loss of information. In our case, there is a loss of information because terms are put in an Euclidean space and because this space has only 2-D. Also, our term map contains both very specific terms and more general terms. From this point of view, terms can be seen as hierarchically organized. However, the hierarchical organization of terms is not visible in our term map. This also causes a loss of information.

Because of the above limitations, a bibliometric map should never be interpreted as a perfectly valid representation of the domain of interest.

Our approach to the interpretation of a bibliometric map is as follows. As pointed out above, a bibliometric map has various limitations. However, the knowledge of an expert in a certain domain will also be limited in various ways. Not only will the knowledge of an expert typically be incomplete, but the knowledge of an expert will also be subject to uncertainty. An expert may have a very detailed and precise knowledge of certain areas within a domain, but his knowledge of other areas may be based only on some vague ideas of a rather uncertain nature. Also, the knowledge of an expert may be partially incorrect. An expert may for example overestimate the importance of his own preferred research topics. Given the limitations of both bibliometric maps and expert knowledge, a bibliometric map can best be seen as a tool that supports experts to improve their knowledge of a certain domain. A bibliometric map can support an expert by extending the expert's (uncertain) knowledge, by decreasing the amount of uncertainty in the expert's knowledge, and by uncovering elements in the expert's knowledge that may not be correct. However, a bibliometric map only makes suggestions to an expert, and the expert should not have blind trust in these suggestions. Instead, the expert should treat the suggestions of a bibliometric map as just one piece of evidence, in addition to other pieces of evidence, such as his own knowledge, the opinions of other experts, and the results of possible other quantitative analyses. 
To determine how much weight one should give to the evidence provided by a bibliometric map, there are two important considerations. First, the larger the amount of data on which a bibliometric map is based, the more confidence one may have in the map. In the case of our term map, we use titles and abstracts of 1,027 articles. This is still a relatively limited amount of data, which means that a careful interpretation of the map is essential. Second, a distinction can be made between a global and a local interpretation of a bibliometric map. In a global interpretation, one looks at the broad areas in a map and how they relate to each other. In a local interpretation, one looks in much more detail, for example at individual terms and their distance from each other. Usually one can have a high degree of confidence in global interpretations. Local interpretations, however, tend to be much more uncertain.

\section{Results}

The term map constructed using the methodology discussed in the previous section is shown in Figs. 1 and 2. These figures display the so-called label view and density view of the map. The label view focuses on the details of the map, while the density view provides a general overview of the map by indicating the relative importance of the various areas in the map. In the density view, the color of an area reflects the number of times the terms located in the area occur in the titles and abstracts of the articles in our data set. The red areas in the density view can be regarded as the most important ones. The terms located in these areas together occur many times in the titles and abstracts of our articles. For a more technical discussion of the density view, we refer to Van Eck and Waltman (2010).

The term map can be explored interactively using the VOSviewer software. To do so, please visit http://www. vosviewer.com/maps/computer_ethics/. ${ }^{3}$ The VOSviewer software has zoom, scroll, and search functionality to facilitate a detailed exploration of the map. The software provides different views, allowing one to focus either on the map's global structure or on its more detailed properties.

Figure 1 shows that each of the three clusters has a more or less central term around which the other terms are positioned. The three central terms are Privacy, Ethics, and Internet. ${ }^{4}$ The font size used to display a term and the size of a term's circle indicate the number of articles in which the term occurs. Hence, in the C\&IE literature, Privacy,

\footnotetext{
3 To run the VOSviewer software, a recent version of Java needs to be installed on your system.

4 The term map shows the word Ethic instead of the word Ethics. This is because our algorithms reduce words to their stem.
}

Ethics, and Internet are the most prominent and the most often discussed topics of the last decade. From now on, we refer to the three clusters as the Privacy cluster, the Ethics cluster, and the Internet cluster. For each cluster, the 30 most occurring terms are listed in Table 1.

The observation that Ethics is one of the most occurring terms in the field should not come as a surprise and needs no further explanation. The prominence of Privacy may be explained by the fact that privacy issues are the epitome of ethical concerns regarding ICT. Privacy issues easily resonate with personal experiences, are close to home, and easily give rise to academic and public debate. In the literature, issues regarding Informed Consent, Security, Safety, Liberty, Free Speech, and so on are often discussed in relation to privacy. Furthermore, the observation that the Internet is the most prominent manifestation of ICT in the C\&IE literature should not come as a surprise either. During the last decade, the Internet has had a profound influence on the life of human beings in all segments of society all around the world. This influence has been widely examined and discussed in the C\&IE literature. We will now analyze each of the three clusters in more detail.

\section{The privacy cluster}

We begin with the privacy cluster (i.e., the green cluster in Fig. 1). After careful analysis, four subtopics can be distinguished in this cluster. The first, although strictly speaking not a subtopic, contains terms that can roughly be seen as synonyms of Privacy, such as Privacy Concern, Information Privacy, Personal Privacy, and Online Privacy. The second subtopic contains ethical terms that are directly relevant for the academic debate on Privacy in relation to ICT, such as Informed Consent, Consent, Security, Safety, Infringement, Personal Information, Free Speech, and Liberty. The third subtopic contains terms that may somehow cause Privacy issues, such as Ubiquitous Computing, Surveillance, Information Flow, Corporation, Information System, Camera, Data Collection, Radio Frequency Identification, Airport, Terrorism, and Workplace. And the fourth subtopic contains terms associated with legal and governmental issues, such as Intellectual Property, Intellectual Property Right, Government, E-Government, Welfare State, Privacy Policy, Privacy Right, Civil Liberty, Property Right, Legislation, Rights, Privacy Protection, and Legal Protection.

Two things are remarkable here. First, privacy issues that are discussed under the heading of Data Protection and Personal Data are separated from discussions about Privacy. This may represents an US-Europe opposition of terminologies and conceptual frameworks, where Privacy is the dominant term in the US and Data Protection is the preferred vocabulary to discuss ethical issues concerning 


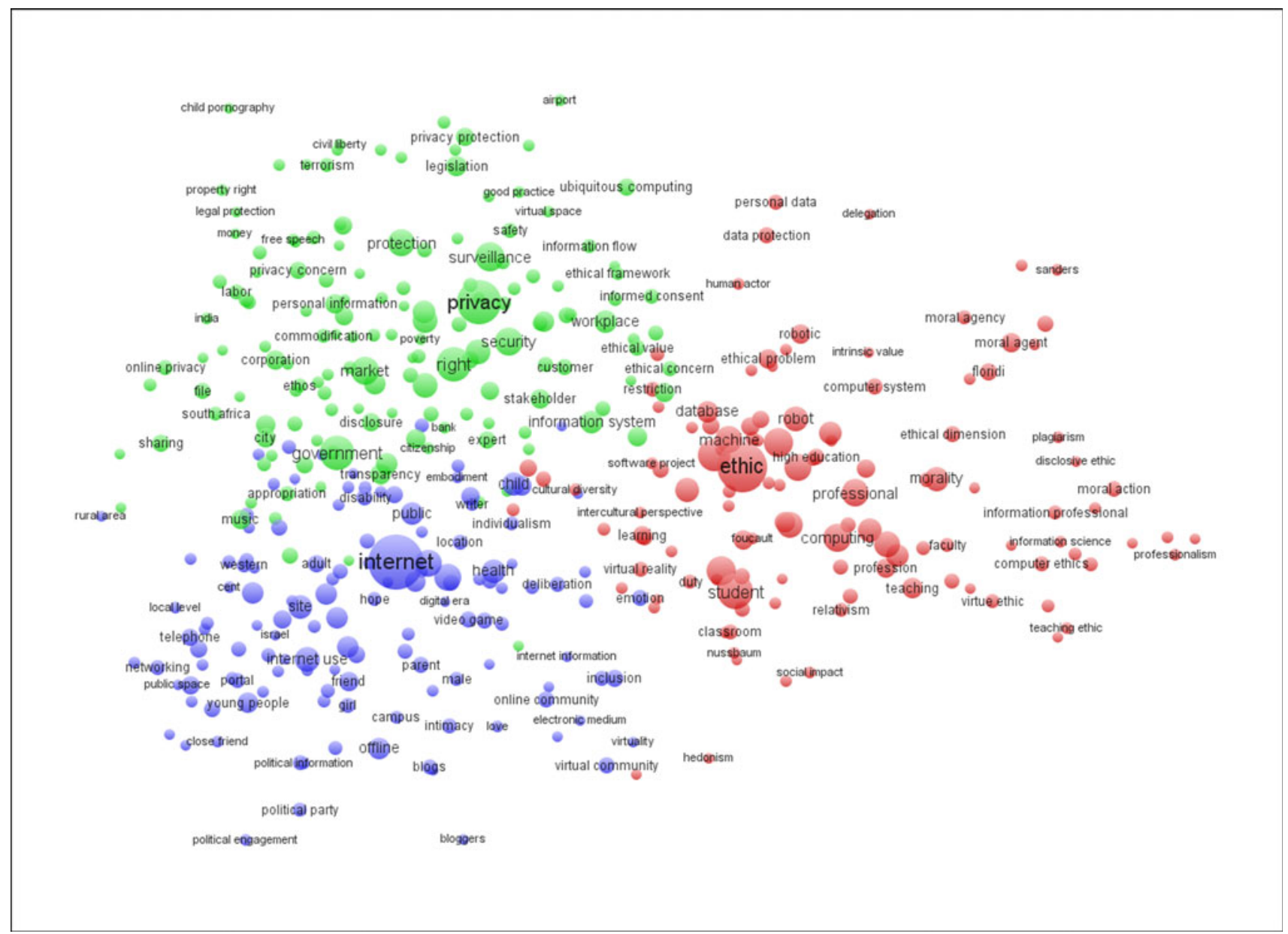

Fig. 1 Map of 400 key terms in C\&IE (label view). The font size used to display a term and the size of a term's circle indicate the number of articles in which the term occurs. The color of a term's circle indicates the cluster to which the term belongs. To avoid

personal information and data flows in Europe. The bibliometric analysis, in line with this opposition of terminologies, puts Personal Information in the privacy cluster and not in the ethics cluster in which Personal Data and Data Protection are put. A second noteworthy feature of the map as depicted in the density view (see Fig. 2) is the way the term Government connects the most prominent themes of Internet and Privacy. This accounts for the fact of a much discussed and widespread fear of an ubiquitous prying E-Government. Furthermore, Government is cast in two roles in the literature, which explains its prominence here: First as a threat to privacy and second as the party that needs to solve privacy problems by means of regulation.

The four subtopics in this cluster (i.e., Privacy and its synonyms, ethical terms that are directly relevant for the academic debate on Privacy, terms that may somehow cause Privacy issues, and terms associated with legal and governmental issues) are closely related and nicely capture the debate on Privacy in relation to ICT. Roughly, these overlapping labels, only a subset of all labels is visible. To explore the map in an interactive way, please visit http://www.vosviewer. com/maps/computer_ethics/

four subtopics can be explained as follows. Governmental institutions may both cause privacy issues by prying on citizens as well as solve privacy issues (which may also be caused by Surveillance, Terrorism, Corporations, and ICTs) by means of legal and policy regulations.

The ethics cluster

Four subtopics can be identified in the ethics cluster (i.e., the red cluster in Fig. 1). First, topics that can roughly be seen as synonyms, or branches, of Ethics, such as Morality, Moral Reasoning, Disclosive Ethics, Moral Action, Virtue Ethics, Good Life, Duty, Moral Status, Moral Responsibility, Ethical Theory, Information Ethics, Computer Ethics, Professional Ethics, and Ethical Decision. Second, topics related to educational issues, such as Teaching, Classroom, Student, Learning, Faculty, High Education, Instructor, Educator, Academic, and Teaching Ethics. Third, topics associated with ICT as a profession and 


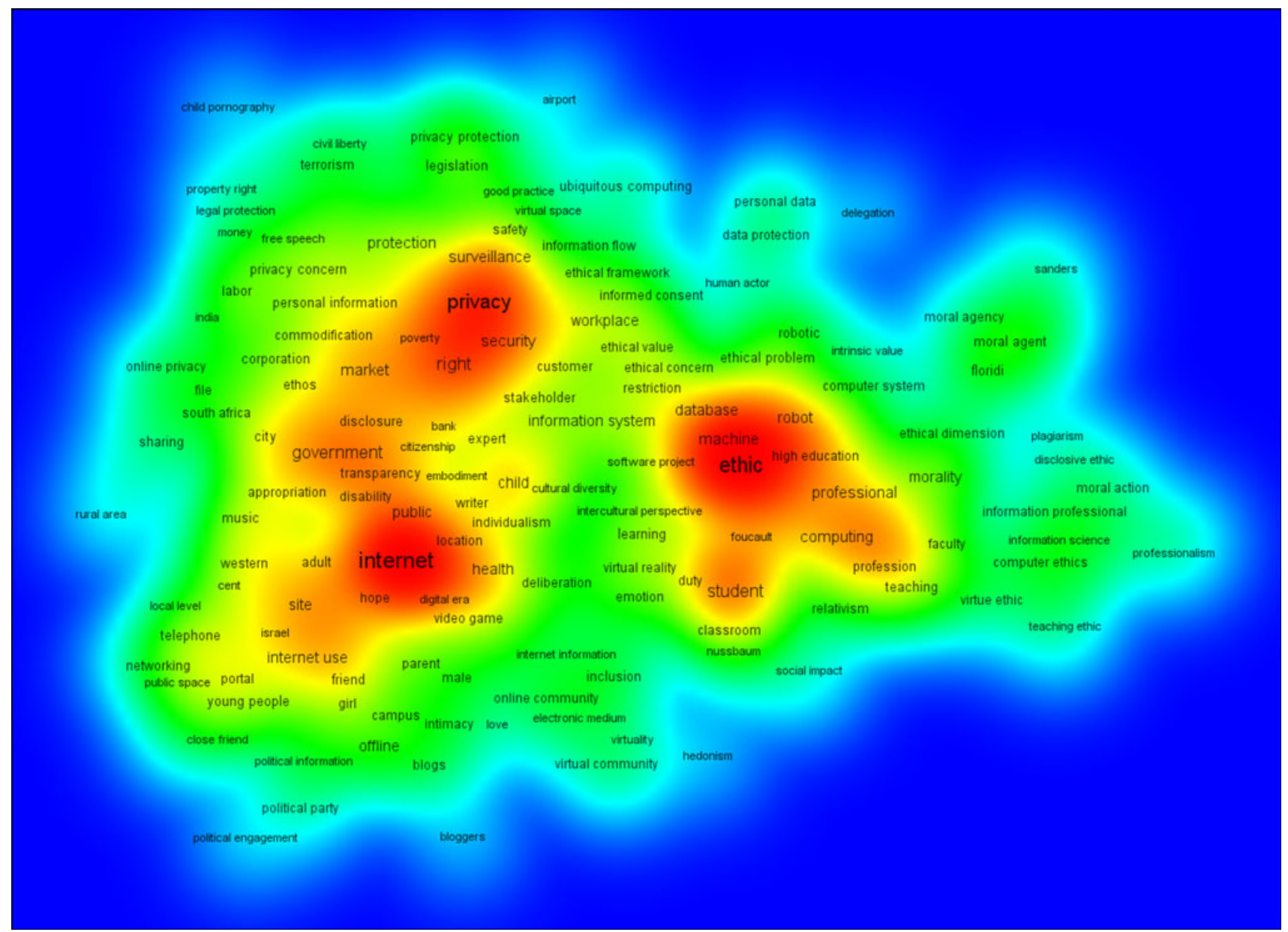

Fig. 2 Map of 400 key terms in C\&IE (density view). Each point in the map has a color that indicates the density of terms at that point. The larger the number of terms in the neighborhood of a point and the larger the total number of occurrences of the neighboring terms, the closer the color of the point is to red. Conversely, the smaller the

academic discipline, such as Professional, Professionalism, Information Professional, Computer Professional, Software Engineering, Computer Science, and Information Science. And fourth, the Ethics cluster contains examples of ICTs, such as Robot, Robotic, Robotics, Artificial Intelligence, Database, Cyborg, Engineering, Infosphere, and Software Agent.

The observation that topics associated with educational issues are presented in the ethics cluster seems consistent with our expectations. There have been substantial discussions about the best approaches to ethics teaching and the use of normative ethical theory for computer scientists, ICT professionals, and computer science students. Some scholars have advocated a virtue ethics approach, character building, development of personal resources in dealing with ethical dilemmas, and methods for exposing students to rich case materials. Others have worked with codes of ethics, checklists, and software to support ethical analysis. number of terms in the neighborhood of a point and the smaller the total number of occurrences of the neighboring terms, the closer the color of the point is to blue. To explore the map in an interactive way, please visit http://www.vosviewer.com/maps/computer_ethics/

And yet others preferred to extend the lessons learned from other areas of applied ethics to the ICT field.

Furthermore, the third and fourth subtopics (i.e., terms associated with ICT as a profession and academic discipline, and examples of ICTs) are closely related and overlap with each other. ICT professionals and computer scientists design and produce the ICTs that cause ethical issues. This observation is consistent with the literature on C\&IE, since the relation between designers of ICTs, the ICTs themselves, and the ethical issues they cause is a widely debated topic in this literature.

Finally, we would like to draw attention to two relatively isolated and coherent sub-clusters within the ethics cluster. One sub-cluster is indicated by terms such as Moral Agent, Moral Agency, and Artificial Agent. The other subcluster is indicated by the terms Personal Data and Data Protection. The relatively isolated position of these two sub-clusters is clearly visible in Fig. 2. 
Table 1 The 30 most occurring terms in the privacy cluster, the ethics cluster, and the internet cluster

\begin{tabular}{|c|c|c|c|c|c|}
\hline Privacy terms & Number of articles & Ethical terms & Number of articles & Internet terms & Number of articles \\
\hline Privacy & 131 & Ethic & 175 & Internet & 228 \\
\hline Government & 74 & Student & 79 & Game & 41 \\
\hline Right & 72 & Responsibility & 63 & Cyberspace & 39 \\
\hline Surveillance & 48 & Human & 49 & Internet use & 32 \\
\hline Market & 42 & University & 47 & Site & 30 \\
\hline Security & 42 & Computing & 42 & Health & 28 \\
\hline Protection & 40 & Machine & 40 & Child & 27 \\
\hline Infrastructure & 34 & Information ethic & 39 & Public & 25 \\
\hline Organisation & 33 & Professional & 38 & Family & 24 \\
\hline Threat & 31 & Computer ethic & 37 & Social network & 23 \\
\hline Information system & 27 & Code & 35 & Email & 22 \\
\hline Intellectual Property & 26 & Robot & 34 & Internet user & 22 \\
\hline Workplace & 25 & Ethics & 30 & Offline & 22 \\
\hline Economy & 24 & Morality & 30 & Expression & 21 \\
\hline Innovation & 23 & Philosophy & 30 & Young people & 21 \\
\hline Device & 21 & Engineering & 26 & Friend & 19 \\
\hline Legislation & 21 & Computer Science & 25 & Patient & 18 \\
\hline Transparency & 21 & Database & 22 & Website & 16 \\
\hline City & 19 & Teaching & 21 & Activist & 15 \\
\hline Information age & 19 & Philosopher & 20 & Interface & 15 \\
\hline Music & 19 & Ethical problem & 19 & Adult & 14 \\
\hline Personal information & 19 & Moral agent & 19 & Campaign & 14 \\
\hline Corporation & 17 & Profession & 19 & Public sphere & 14 \\
\hline Ethical aspect & 17 & Computer technology & 17 & Social interaction & 14 \\
\hline Ethical implication & 17 & Robotic & 17 & Telephone & 14 \\
\hline Stakeholder & 17 & Learning & 16 & Inclusion & 13 \\
\hline Usage & 17 & Human being & 15 & Computer game & 12 \\
\hline Disclosure & 15 & Information ethics & 15 & Connectivity & 12 \\
\hline Intellectual property right & 15 & Artificial intelligence & 14 & Online community & 12 \\
\hline Personal privacy & 15 & Ethical theory & 14 & Virtual community & 12 \\
\hline
\end{tabular}

The internet cluster

The Internet cluster (i.e., the blue cluster in Fig. 1) accommodates much of the issues that have become associated with Internet use in the last decade in the academic as well as the public debate on the Internet. Two categories of subtopics can be distinguished. First, terms that are somehow instantiations of the Internet, such as Site, Blog, Website, Social Networking Site, Virtual world, Virtuality, Online Game, Cyberspace, Avatar, Online Community, Interface, Electronic Medium, Internet Information, Portal, and Online Environment. And second, terms that are associated with social issues in relation to the Internet, such as Health, Inclusion, Social Ty, Political Participation, Parent, Intimacy, Race, Social Interaction, Gender Equality, Social Capital, Social Network, Friend, Protest, Democratic Potential, Individualism, Human Relationship, and Public Access.
The fact that these two categories (i.e., instantiations of the Internet and social issues in relation to the Internet) are put in the same cluster seems logical, since the first exerts an influence over the second, which is at the heart of the C\&IE literature.

\section{Conclusions}

We have presented a bibliometric mapping analysis of the field of C\&IE. A representative data set of titles and abstracts of over thousand articles published in leading journals and conference proceedings in the C\&IE field was constructed and subsequently analyzed with a computer program called VOSviewer. The term map that was produced contains three clusters, namely a Privacy cluster, an Ethics cluster, and an Internet cluster. It can be concluded that privacy issues related to the Internet are the most 
prominent topic in the C\&IE literature of the last decade. Other important topics are privacy in relation to governmental and legal institutions, educational issues in ethics teaching, the relation between designers of ICTs, the ICTs themselves, and the ethical issues they cause, and social issues in relation to the Internet. Another important but somewhat separate topic concerns the moral agency of autonomous agents. Discussions about identity in relation to ICT and on-line environments seem to be less prominent in the period studied and may possibly constitute a recent conceptual and terminological turn in debates about privacy and data protection. This may be shown by follow-up research.

As we have argued, a bibliometric map provides a tool that supports experts to improve their knowledge of the field in which they are active. A bibliometric map makes all kinds of suggestions. These suggestions may confirm or contradict an expert's ideas on the state of his field. They may also assist an expert in developing new ideas. However, even with the help of a bibliometric map, complete consensus among experts seems unlikely to emerge. Most probably, different experts will give somewhat different interpretations to a bibliometric map. For this point of view, it is always good to have the opinions of multiple experts. We therefore invite anyone with an interest in the C\&IE field to explore our term map in more detail and to interpret the map from his own perspective. The map is accessible

at http://www.vosviewer.com/maps/computer_ethics/, where it can be explored using the VOSviewer software.

The study reported in this paper can be seen as a first step towards a dynamic analysis of the C\&IE field. Studies similar to ours can be performed in the future and may then indicate the developments that are going on in the C\&IE field. For example, concepts may gain or lose attention over time, relations between concept may change, and, at a more global level, topics may disappear or new topics may emerge. Furthermore, instead of term maps based on term co-occurrences, other types of bibliometric maps may be used. One may for example use maps of documents, authors, or journals. Instead of term co-occurrences, one may use co-citations or co-authorships to measure relations between items. Future studies may also use other types of data, such as books, course materials, and reading lists.

Acknowledgments We would like to thank Ludo Waltman and three anonymous referees for their comments. These comments have substantially improved the paper. This research is part of the ETICA (Ethical Issues of Emerging ICT Applications) project, an international and interdisciplinary research project funded by the Science in Society program of the 7th Framework Programme of the European Commission. The project identifies emerging ICTs, their potential application areas, and ethical issues to which they may give rise. Based on this, the project aims to develop policy recommendations for the European Commission.
Open Access This article is distributed under the terms of the Creative Commons Attribution Noncommercial License which permits any noncommercial use, distribution, and reproduction in any medium, provided the original author(s) and source are credited.

\section{References}

Borg, I., \& Groenen, P. J. F. (2005). Modern multidimensional scaling (2nd ed.). Berlin: Springer.

Brey, P., \& Søraker, J. H. (2009). Philosophy of computing and information technology. In A. Meijers (Ed.), Philosophy of technology and engineering sciences (pp. 1341-1408). USA: Elsevier.

Buchanan, E. A. (1999). An overview of information ethics issues in a world-wide context. Ethics and Information Technology, 3(1), 193-201.

Bynum, T. (2008). Computer and information ethics. In Stanford encyclopedia of philosophy. Retrieved February 25, 2010, from http://plato.stanford.edu/entries/ethics-computer/.

Callon, M., Law, J., \& Rip, A. (Eds.). (1986). Mapping the dynamics of science and technology. USA: MacMillan Press.

Floridi, L. (Ed.). (2010). The Cambridge handbook of information and computer ethics. Cambridge: Cambridge University Press.

Johnson, D. (1985). Computer ethics (1st ed.). NJ, USA: Prentice Hall.

Johnson, D. (2008). Computer ethics (3rd ed.). NJ, USA: Prentice Hall.

Moor, J. H. (1985). What is computer ethics? Metaphilosophy, 16(4), $266-275$

Newman, M. E. J., \& Girvan, M. (2004). Finding and evaluating community structure in networks. Physical Review E, 69(2), 026113.

Ocholla, D. N., Onyancha, O. B., \& Britz, J. (2010). Can information ethics be conceptualized by using the core/periphery model? Journal of Informetrics, 4(4), 492-502.

Peters, H. P. F., \& Van Raan, A. F. J. (1993). Co-word-based science maps of chemical engineering. Part I: Representations by direct multidimensional scaling. Research Policy, 22(1), 23-45.

Stahl, B. C., Heersmink, R., Goujon, P., Flick, C., Van den Hoven, J., Wakunuma, K. J., et al. (2010). Identifying the ethics of emerging information and communication technologies: An essay on issues, concepts and method. International Journal of Technoethics, 1(4), 20-38.

Tavani, H., \& Himma, K. (Eds.). (2008). The handbook of information and computer ethics. NJ, USA: Wiley.

Tijssen, R. J. W., \& Van Raan, A. F. J. (1989). Mapping co-word structures: A comparison of multidimensional scaling and LEXIMAPPE. Scientometrics, 15(3-4), 283-295.

Van den Hoven, J., \& Weckert, J. (Eds.). (2008). Information technology and moral philosophy. Cambridge: Cambridge University Press.

Van Eck, N. J., \& Waltman, L. (2010). Software survey: VOSviewer, a computer program for bibliometric mapping. Scientometrics, 84(2), 523-538.

Van Eck, N. J., Waltman, L., Dekker, R., \& Van den Berg, J. (2010a). A comparison of two techniques for bibliometric mapping: Multidimensional scaling and VOS. Journal of the American Society for Information Science and Technology, 61(12), 2405-2416.

Van Eck, N. J., Waltman, L., Noyons, E. C. M., \& Buter, R. K. (2010b). Automatic term identification for bibliometric mapping. Scientometrics, 82(3), 581-596.

Waltman, L., Van Eck, N. J., \& Noyons, E. C. M. (2010). An unified approach to mapping and clustering of bibliometric networks. Journal of Informetrics, 4(4), 629-635. 